\title{
Wie sich Adipositas, Diabetes und Co. in der Schwangerschaft auswirken
}

\author{
Bei 714.547 Geburten in Deutschland im Jahr 2015 waren 6.579 (0,92\%) der Mütter schon präkon- \\ zeptionell an Diabetes erkrankt. Die enge Verzahnung der Stoffwechselerkrankung mit Überge- \\ wicht und wie sie sich auf Mutter und Kind auswirkt war Thema beim DiabetesUpdate in Mainz.
}

Auch bei Frauen im reproduktionsfähigen Alter nimmt Adipositas h zu, erklärte Dr. Helmut Kleinwechter vom diabetologikum Kiel. Die Sorgen betreffen dabei zwei Generationen: die Mütter mit ihrem erhöhten Risiko für Stoffwechselkrankheiten und kardiovaskuläre Morbidität und die Kinder, die schon bei Geburt das Risiko für Adipositas mit auf den Weg bekommen.

Adipositas in der Frühschwangerschaft kann mit erhöhten Risiken für Totgeburt (fetaler Tod ab 28. SSW) und gesteigerter kindlicher Mortalität (im 1. Lebensjahr) einhergehen. Dies hat eine schwedische Fall-Kontroll-Studie gezeigt [1]. Verglichen mit einer Kontrollpopulation (Body Mass Index (BMI) 18,5-24,9) stieg das Risiko für Totgeburt bei Frauen mit BMI von 25-29,9 auf eine OR von 1,51 an, bei BMI von 30-34,9 auf OR 1,77 und bei BMI $\geq 35$ auf OR 3,16. Die geeignete Zeit für Ratschläge zur Lebensstilveränderung sei postpartal, so Kleinwechter, um das Gewicht noch vor der nächsten Schwangerschaft zu reduzieren. Die Risiko-Kommunikation in der Schwangerschaft sei psychologisch schwierig. Um Kalorien zu sparen, ist der Ersatz von $\mathrm{Zu}-$ cker durch künstliche Süßstoffe offenbar kein guter Weg. Erstmals wurde in einer humanmedizinischen Studie ein Zusammenhang zwischen dem täglichen Süßstoffkonsum (künstliche Süße, Zuckerersatzstoffe) in der Schwangerschaft und einem gesteigerten Risiko für einen erhöhten kindlichen BMI gezeigt [2].

\section{Gewichtszunahme in der Schwangerschaft}

Schwangere Frauen sollten nicht zu stark zunehmen. Beobachtungsstudien zeigten eine Assoziation von exzessiver Gewichtszunahme mit höherem Geburtsgewicht der Kinder und höherem (BMI) in der Kindheit. Mütter mit starker Gewichtszunahme in der Schwangerschaft hatten später Töchter, die dreimal öfter in ihren 40er Jahren übergewichtig/adipös waren, dies v.a. bei Müttern mit schon präkonzeptionellem Übergewicht (OR 3,4, RR 1,5) [3]. Als State of the Art für die empfohlene Gewichtszunahme in der Schwangerschaft gilt die IOM-Leitlinie (Institute of Medicine), die 1970, 1990 und 2009 publiziert wurde und

\begin{tabular}{ll} 
T1 IOM-Leitlinie zur Gewichtszunahme in der Schwangerschaft \\
(publiziert 1970, 1990, 2000) \\
\hline Präkonzeptioneller BMI (kg/m2) & Ziel Geburt (kg) \\
$<18,5$ & $12,7-18,2$ \\
$18,5-24,9$ & $11,5-16$ \\
$25,0-29,9$ & $7-11,4$ \\
30 & $5-9$ \\
Quelle: Diabetes Update 2017 & \\
\hline
\end{tabular}

weltweit angewendet wird ( $\triangleright$ Tab. 1). Wegen der großen Variabilität der länderspezifischen Erhebungen bieten die Angaben aber nur eine grobe Orientierung, so Kleinwechter. Die höchste Evidenz bei präkonzeptionell normgewichtigen, gesunden Schwangeren sieht er für den Korridor von 8,7-20,2 kg bis zur 40. Schwangerschaftswoche. Das sei viel breiter als nach der IOM-Leitlinie.

\section{Präeklampsierisiko und Fragen zum Stillen}

Schwangere mit präkonzeptionellem Diabetes und Übergewicht sind eine Hochrisikogruppe, die im Vergleich zu nicht diabetischen Schwangeren 3,7-mal häufiger eine Präeklampsie erleidet. Präventiv kann ASS 100 mg/Tag ab der 12.-16. SSW eingenommen werden [4]. Typ-1-Diabetes ist per se ein stärkerer Risikofaktor für Präeklampsie als der BMI [5]. Pathophysiologisch haben Adipositas und Präeklampsie einiges gemeinsam: z.B. subklinische Inflammation, Insulinresistenz und Lipotoxizität.

Typ-1-Diabetikerinnen müssen beim Stillen keine Sorge vor Hypoglykämien haben, ergab eine australische Untersuchung beim Vergleich von stillenden Müttern mit Typ-1-Diabetes $(n=8)$ mit flaschenfütternden Müttern $(n=8)$ [6]. Es wurden die Verläufe der Gewebeglukose bis zu 6 Tage in einem Zeitraum von 2-4 Monaten post partum gemessen. Stillende Frauen nahmen einen größeren Anteil von Kohlehydraten zu sich als flaschenfütternde, bei vergleichbarer Insulingabe. Jedoch waren hohe Glukosewerte und deren Streubreite bei letzteren ausgeprägter. Niedrige Glukosewerte waren nach dem Stillen häufiger als vor oder während dessen. Hypoglykämien < $72 \mathrm{mg} / \mathrm{dl}$ traten bei $14 \%$ der Mütter innerhalb von 3 Std. nach Stillen auf, und zwar abhängig vom letzten Essen und der letzten Dosis eines kurz wirksamen Insulins. Nach einem Standardfrühstück war die Fläche unter der Kurve bei Stillenden positiv, wenn sie nicht in der ersten Stunde nach dem Essen mit dem Stillen begonnen hatten und negativ, wenn das Stillen innerhalb von 30 Minuten nach einer Mahlzeit gestartet wurde. Somit gab es keine Unterschiede bei Hypoglykämien zwischen stillenden und flaschenfütternden Müttern. Stillen senkt zwar die mütterlichen Glukose, führte aber bei den meisten Stillepisoden nicht zur Hypoglykämie. Dagmar Jäger-Becker



Quelle: DiabetesUpdate 2017, Mainz, 18. März 2017 